\title{
An Automatic Control Method of Foam Spraying Glue Machine based on DMC Yu-An HE ${ }^{a,{ }^{*}}$, Tian CHEN ${ }^{b}$ \\ College of Engineering, Shanghai Polytechnic University, Shanghai, China \\ ayahe@sspu.edu.cn, b497309069@qq.com \\ ${ }^{*}$ Corresponding author
}

Keywords: Digital Motion Control (DMC), Automatic Spray Machine, Foam Mattress, Spray Glue.

\begin{abstract}
Memory foam is a kind of Polyether polyurethane foam which possesses low resilience mechanical properties. The foam mattresses are generally consisted of two to six layers foam, and glued by workers manually with Acrylic glue. A DMC (Digital Motion Control) based control system of automatic spraying glue of foam mattress is proposed. The Galil DMC is utilized for the motion control of three axes. By using Visual Basic and Galil motion control codes, the software of control system are programmed. The required motion routes of two spray guns will be realized on the mattress. The practice shows that the system runs reliably, the production efficiency shows obviously, and the working environment of spray workers has been greatly improved.
\end{abstract}

\section{Introduction}

With the development of automation technology, computer technology and modern information technology, and the continuing growth of labor costs, market demand for high precision and automatic equipment is also growing.

Elastic memory foam mattress can improve human comfort and sleep quality, which is more and more popular with people. In order to increase the permeability, production is the number of layers of foam mattresses together, resulting in different layer and different thickness of mattress products. At present, the commonly method is to spray glue to cushions by the manual spray gun, and to cover another pieces of cushions by artificial way in the above. The working environment of workers is poor, uneven glue, glue waste, labor intensity, low labor productivity, and low degree of automation, high labor costs. So, it is very significant to develop a set of automatic gluing and automatic promotion foam and covering glue automation equipment to improve the working environment of workers, reduce the labor intensity of workers, and increase production efficiency $[1,2]$.

Through the project design and development, we can design a set of automatic spray glue equipment for foam mattress. According to the requirements, this device can complete different size (length and width), multiple layers of foam mattresses by automatic spray glue, it can realize automatic spray glue with spray chamber enclosed, and realize automatic counting of foam mattress production, which can constitute the foam mattress production line with automatic spray glue machine (AGM), mattress loading and unloading robot.

\section{Structural Design and Working principle of AGM}

Automatic spray glue machine (AGM) is mainly composed of spray chamber, conveyor, nozzle, $\mathrm{X}, \mathrm{Y}, \mathrm{Z}$ three directions of linear module, fog absorption device. It is shown in Figure 1.

Spray chamber has two nozzles, $\mathrm{X}, \mathrm{Y}, \mathrm{Z}$ direction linear module, fog absorption system and an outside cover. $\mathrm{X}$ direction has two linear modules, which can realize synchronous movement by a long shaft connection. Y direction linear module is consist of two segments of the contrary rotating ball screw, $\mathrm{Z}$ direction linear module drives the nozzles to the synchronous movement at both direction, and keeping screw rotating at the same time.

The working environment of workers improved significantly, because spray chamber can prevent glue and fog out of the chamber. Nozzles can complete the required spraying glue line through the 
movement of the $\mathrm{X}$ axis and $\mathrm{Y}$ axis and conveyer belt, initial position is located in the middle of spray nozzle, which can further prevent spray glue on both sides of the mist from the spray of cushion import and export overflow (both sides will pack sealing curtain).

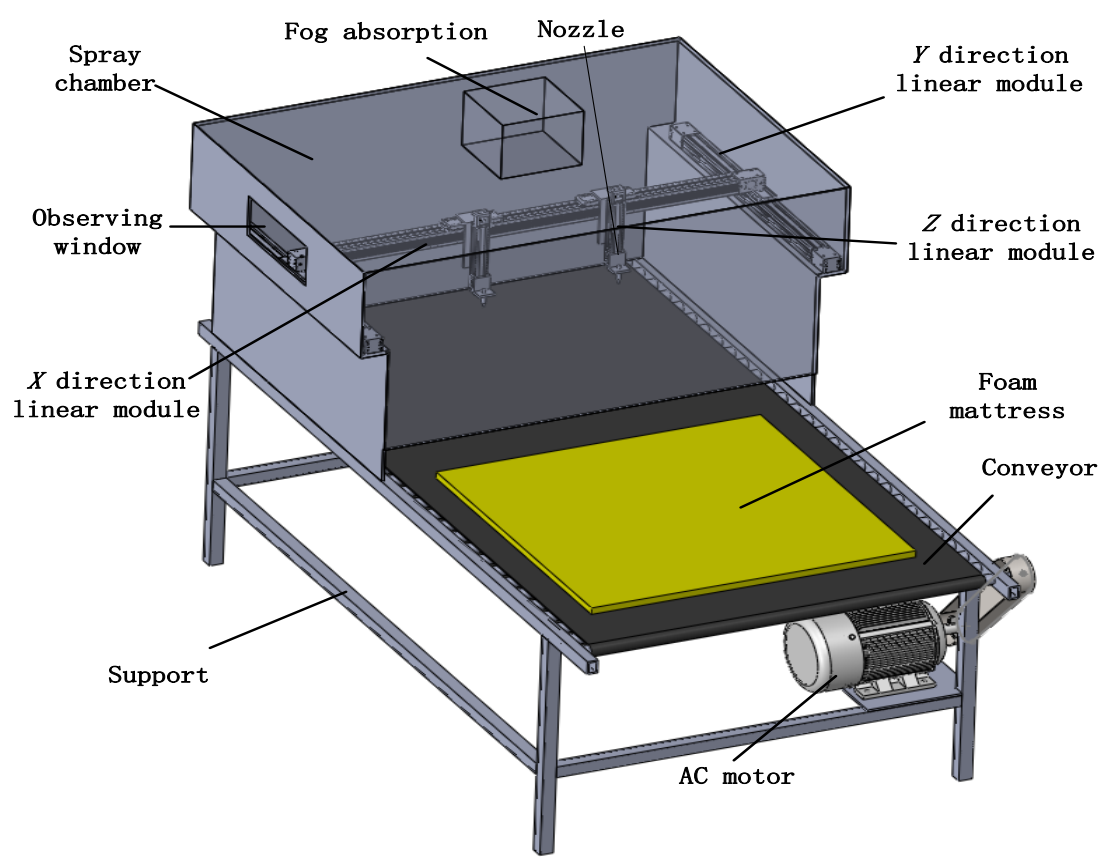

Fig. 1 Structure diagram of automatic glue machine.

Conveyor belt is made up of drum, roller, belt and frame and etc, driving drum is located in the right side, which is driven by a three-phase motor. Automatic spraying glue machine is also equipped with suction device, which can control the humidity of spray chamber, and prevent the fog too thick. Working principle of automatic spray machine: Three axes control the motion, one axis control nozzle, $\mathrm{X}$ and $\mathrm{Y}$ direction of the linear module ensure reasonable spray glue route, the $\mathrm{Z}$ axis is used to adjust the nozzle and the distance of the mattresses, making the different thickness of cushion sprinkler agree with cushions spacing and cooperate with axis which controls the nozzle to control the amount of glue, in order to ensure the quality of spraying glue and spraying glue area. The process route is spraying on the head of mattresses firstly, and the secondly on both sides of the mattress and the tail, finally, on the centre of the mattress.

Controlling two nozzles to run laterally to spray the head by the $\mathrm{X}$ direction linear module, and spray the both side by $\mathrm{Y}$ direction linear modules, then controlling two nozzles to run interiorly to spray the tail by $\mathrm{X}$ direction linear modules again. According to the spray glue machine working process, the nozzle spraying routes for one spray glue layer of mattress are shown in Fig. 2 (a) to Fig.2 (d). 


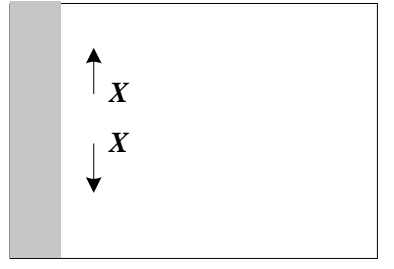

(a) Step one: Y axis move, spray the head



(c) Step three: $Y$ axis move, spray the tail

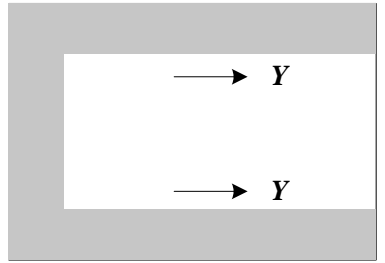

(b) Step two: X axis move, spray two sides

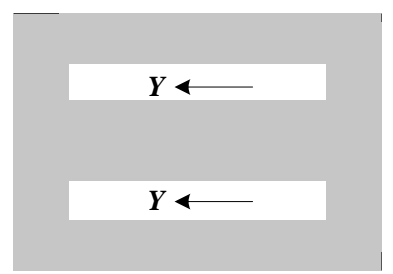

(d) Step four: X axis move reverse, spray the middle

Fig. 2 The route and process of spray nozzles moving.

\section{Control System Hardware Based on DMC}

DMC-B140 is four axes motion controller for economical and independent Ethernet controller itself provides four-axis motor control interface and the general $\mathrm{I} / \mathrm{O}$ signal interface and the user program and data storage space.

DMC-B140 is a comprehensive controller which can work independently and use communication interface with other controllers and man-machine interface in order to achieve more complex function and more convenient operation. In addition, DMC-B140 provides two kinds of communication channel (the highest serial transmission rate 115.2 RS-232 and 10/100 m Ethernet), it provides more than 200 instruction and support conditional logic judgment and shaft. it also provides the origin switch and both ends of travel limit switch and encoder feedback for each connected to the motor movement, Controller provides eight general purpose digital input and four general digital output which can be connected to deal with external relay and sensor.

The control system is a double CPU control system which adopts PC and DMC-B140 motion control card. It can complete the actual movement of position, velocity, acceleration, servo and stepper control and other I/O control. The device can realize the motion controller and communicate with PC by using RS-232 communication channel, In addition to the PC and motion controller, system also includes the stepper motor, the drive, intermediate connecting plate, the I/O interface board, DC24V power supply models, limit switch, relay models, buttons, etc. System hardware structure is shown in Figure 3. In the control system, DMC motion controller and the motor drives work requires an external power supply $24 \mathrm{v}$.

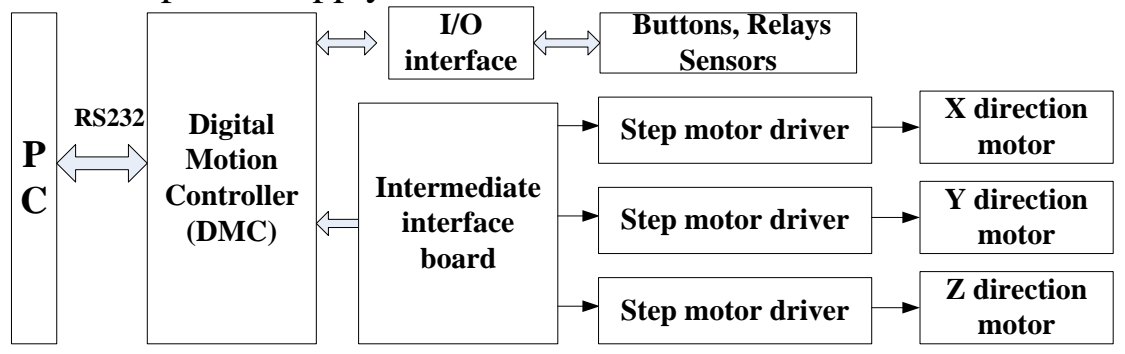

Fig. 3 The system hardware block diagram.

\section{Control System Software Based on DMC}

DMC-B140 motion controller supports user using DMC WIN32 Galil Windows API and function to use $\mathrm{VB}, \mathrm{VC}, \mathrm{VS}$, in order to meet the requirements of high-level language such as $\mathrm{C} / \mathrm{C}++$ applications. 
Software design and implementation of automatic spray glue system is the core part of the whole system, the control system program flow chart is shown in Figure 4. In order to obtain better manmachine interface, we can use Visual Basic user operating interface. The interface includes the process of the parameter setting and work status display when automatic gluing device is in the working. Interface can set the length and width of the mattress, mattress layer numbers, the thickness of each layer of mat, three axis movement speed, etc. It also can realize production count, quality control and safety alarm, etc.

In the whole system design, first call DMCOPen() function to establish the connection between the PC and motion controller, and then through the DMCCommand(), DMCDownloadFile(), and DMCDownloadFromBuffer() function to send instructions to the motion controller. After the command is sent to the motion controller, the $X Q, B G$ control instructions to drive the movement, and then complete the control operation. It can set pause point of the execution of a program through $A M$, the $A S$ and $A D, W T$ instruction, in order to relieve the main PC operation pressure, and improve the operation efficiency of the system.

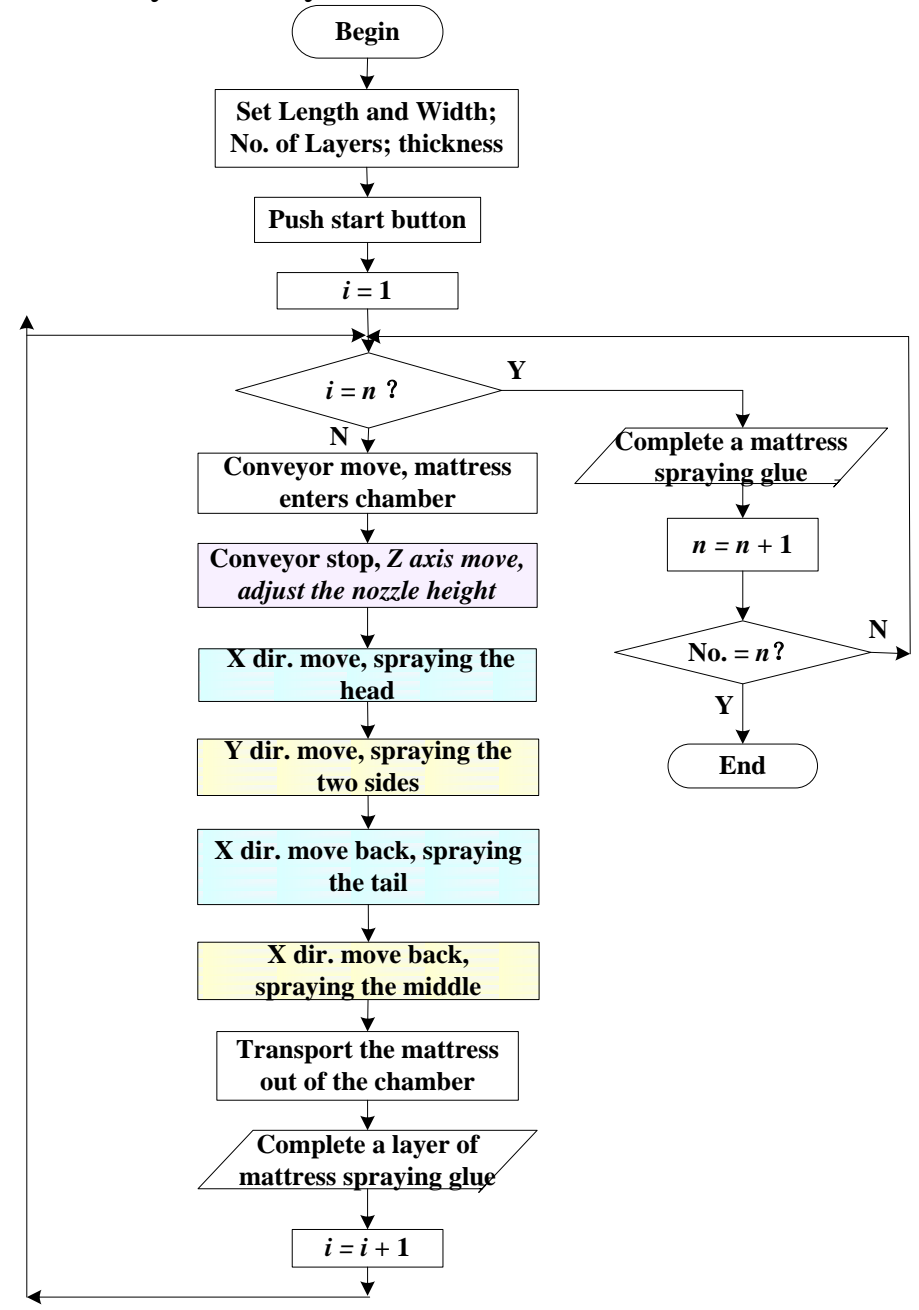

Fig. 4 The procedure flow charts of control system.

\section{Conclusion}

Foam mattress automatic spraying glue machine is the enterprise actual engineering application project.

On the basis of sufficient investigation and research, firstly, we design and optimize automatic spraying glue machine mechanical structure by using the 3D CAD software. We design the control system of automatic spray glue machine parts of hardware and software, the project adopts PC and DMC motion controller of double CPU as the control core, it's operation speed, low cost, stable and reliable. In VB development environment, we design the software with DMC motion control 
instruction, and the system has friendly man-machine interface and easy operation. the new type of automatic spray equipment that we research and develop in this topic can greatly improve the working environment, and increase spray glue production efficiency greatly, the workers' labor intensity is reduced, the enterprise core competitiveness improved. The control method can be widely applied to mechanical equipment automation transformation, which is based on DMC, and it has a certain application promotion value.

\section{References}

[1]G. H. Shao, Automatic spraying glue machine motion control system. J. Servo control. 2011(3): 84-86.

[2]W. Chen, Z. C. Huang, C. Y. Zhou, The development of the automatic spray glue machine and its control system. J. Mechanical engineer. 2012(8): 72-74.

[3]Q. H. Pei,Q. X. Wang, J. G. Yang, Image measuring instrument motion control system design based on the GALIL, J. Mechanical engineer. 2009(7):59-60.

[4]Y. B. Zhang, Z. Y. Xie, Z. M. Wang, Three-axis motion control card of the software system development research based on DMC1380, J. Shan Dong Science. 2013, 23(2): 38-40.

[5]Galil Application Notes - DMC Programming information on http://www.galilmc.com/. 